\title{
How MSF is mapping the world's medical emergency
} zones

Clarification-A quote used in this Feature (BMJ

2014;349:g7540, doi:10.1136/bmj.g7540) incorrectly suggests that the mapping work carried out to support the Ebola crisis was done entirely by the Missing Maps project. In fact, the
Missing Maps project assisted the Humanitarian OpenStreetMap Team with this work.

Cite this as: BMJ 2014;349:g7644

๑ BMJ Publishing Group Ltd 2014 